\title{
Experience using propranolol for the management of supraventricular tachycardia in patients younger than 1 year
}

\author{
Eliana S. Nicastro, M.D. ${ }^{a}$, María G. Majdalani, M.D. ${ }^{a, b}$, Mauricio S. Abello, M.D. ${ }^{b}$, David G. Doiny, M.D. ${ }^{b}$, \\ Estela C. Falconi, M.D. ${ }^{b}$, Carlos J. Díaz, M.D. ${ }^{b}$ and José M. Moltedo, M.D. ${ }^{a, b}$
}

\begin{abstract}
Objective. To assess the results and adverse events of propranolol therapy in infants younger than 1 year with supraventricular tachycardia.

Population and methods. Infants younger than 1 year with documented supraventricular tachycardia who received oral treatment and prophylaxis with propranolol. Sex and age, associated congenital heart disease, ventricular preexcitation in the base line electrocardiogram, on-treatment recurrence, and adverse events were analyzed.

Results. A total of 107 patients were identified. The first supraventricular tachycardia event occurred at a median age of 190 days. Associated congenital heart disease was observed in 10 patients. Ventricular preexcitation in the baseline electrocardiogram was detected in $23.3 \%$. Propranolol dose ranged from 2 to $5 \mathrm{mg} / \mathrm{kg} /$ day. On-treatment recurrence was observed in $30.8 \%$. Medication was discontinued in 2 patients due to severe adverse events.

Conclusion. Propranolol prevented recurrence in $70 \%$ of cases. It was discontinued in 2 patients due to severe adverse events. Keywords: pediatrics, supraventricular tachycardia, antiarrhythmics, propranolol.
\end{abstract}

http: / / dx.doi.org/10.5546/ aap.2020.eng.273

To cite: Nicastro ES, Majdalani MG, Abello MS, Doiny DG, et al. Experience using propranolol for the management of supraventricular tachycardia in patients younger than 1 year. Arch Argent Pediatr 2020;118(4):273-276. a. Clínica y Maternidad Suizo Argentina, Autonomous City of Buenos Aires, Argentina.

b. Sanatorio Finochietto, Autonomous City of Buenos Aires, Argentina.

E-mail address:

Eliana S. Nicastro, M.D.: nicastro.eliana@gmail.com

Funding: None.

Conflict of interest: None.

Received: 4-8-2019

Accepted: 12-26-2019

\section{INTRODUCTION}

Supraventricular tachycardia (SVT) is defined as any tachyarrhythmia that originates above the His bundle. ${ }^{1}$ Neonates and infants have a high risk for inadvertent SVT events and development of cardiomyopathy and ventricular dysfunction, so drug therapy is recommended for resolution and prevention. ${ }^{2-5}$ SVT debut is more common in the first year of life, with a higher incidence in the neonatal period and between the third and fourth month of life. ${ }^{3}$ Propranolol is a beta-adrenergic receptor blocker used as a first-line treatment drug for the management and prevention of recurrence in neonates and infants. ${ }^{2,4}$ The most common adverse events associated with propranolol use include sinus bradycardia, dizziness, hypotension, and hypoglycemia, among others. ${ }^{2-4}$

\section{OBJECTIVE}

To assess the results and incidence of adverse events of oral propranolol therapy in infants younger than 1 year with SVT.

\section{POPULATION AND METHODS}

This was an observational and descriptive study based on the analysis of the outpatient office database of a Department of Pediatric Electrophysiology in the Autonomous City of Buenos Aires. The study period was between August 2001 and January 2018.

Patients younger than 1 year with electrocardiogram-documented SVT events and spontaneous or drug reversion and who received oral propranolol (4 mg/mL oral solution or cachets) as control and prophylaxis therapy for a year were identified. Propranolol dose was $3 \mathrm{mg} /$ $\mathrm{kg}$ / day administered as 4 daily doses for the first 3 months and then changed to 3 daily doses in a range of $2-5 \mathrm{mg} / \mathrm{kg} /$ day as adjusted based on patient weight during follow-up.

Patients with ectopic atrial tachycardia, atrial flutter without any other associated tachycardia, and those who were lost to follow-up were excluded. The following were analyzed: sex and age at the time of SVT diagnosis, presence 
of associated congenital heart disease (CHD), ventricular preexcitation in the baseline electrocardiogram (ECG), onset of severe adverse events, on-treatment recurrent SVT events (in the first year of the first SVT event).

Treatment started while patients were hospitalized and under continuous monitoring of their heart rate and drug adverse events onset. A color Doppler echocardiogram was done in all patients to assess ventricular function and rule out an associated structural heart disease.

Follow-up was done in the outpatient offices with an ECG and a Holter ECG in the 1-year period after the event. Propranolol power to prevent SVT recurrence was assessed, and cardiomyopathy and ventricular dysfunction were prevented. The drug was discontinued if symptomatic adverse events occurred.

Numerical data were described as percentage and median and / or mean \pm 2 standard deviations (SD). A two-tailed $t$ test for groups with different variances was done to assess the difference between the groups with and without ontreatment recurrent SVT events. Pearson $\chi^{2}$ test was used to analyze the frequency of recurrence between the group with a normal ECG and that with ventricular preexcitation. The probability of recurrence was assessed with the Kaplan-Meier test.

\section{RESULTS}

A total of 107 patients (62 males) diagnosed with SVT were identified. The first event occurred at a median age of 190 days (range: 1-365). Ten patients had an associated CHD (6 had ventricular septal defect). None had ventricular dysfunction at diagnosis. Ventricular preexcitation in the baseline ECG was detected in 25 patients $(23.3 \%)$.

Propranolol dose ranged from 2 to $5 \mathrm{mg} /$ $\mathrm{kg} /$ day, and the mean dose was $3 \pm 0.5 \mathrm{mg} / \mathrm{kg} /$ day. On-treatment recurrent SVT was observed in 33 patients $(30.8 \%)$ during follow-up. The Kaplan-Meier curve showed that the probability of recurrence after one month of treatment was $23.78 \%$ (95\% confidence interval [CI]: 16.4933.58) and, at 12 months, $29.2 \%$ (95\% CI: 22.1639.44) (Figure 1). Patients with on-treatment recurrence were divided for analysis into those with ventricular preexcitation in the baseline ECG ( $\mathrm{n}=8,24.2 \%$ of patients with recurrence), and those with normal baseline ECG $(\mathrm{n}=25$, $75.7 \%$ of patients with recurrence). In relation to the baseline ECG, the difference in the frequency of recurrence between the group with a normal baseline ECG and the group with ventricular preexcitation was not significant $(\mathrm{p}=0.886)$ as per Pearson $\chi^{2}$ test.

Two patients had to discontinue propranolol due to adverse events. Both had been admitted

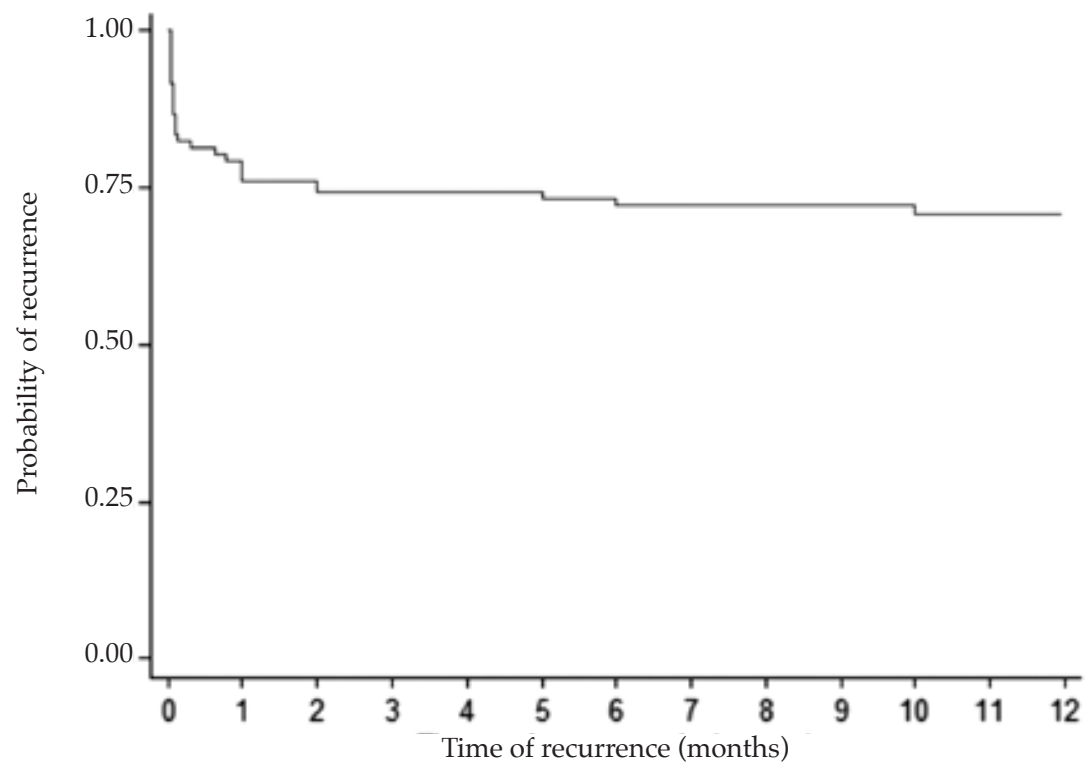


through the Emergency Department with sensory deficit and vomiting, and symptomatic hypoglycemia (blood glucose below $50 \mathrm{mg} / \mathrm{dL}$ ) was detected in the context of concurrent viral infections. No patient in this population died in the follow-up period.

\section{DISCUSSION}

Propranolol use as an antiarrhythmic agent for the prevention of SVT events in neonates and infants has increased in recent years. However, few randomized studies with sufficient statistical power to assess propranolol use in this age group have been conducted. ${ }^{4,5-7}$

In this group of patients, the mean dose was $3 \mathrm{mg} / \mathrm{kg} /$ day, which managed to prevent recurrent events in the first year of treatment in most patients. Such control rate without on-treatment recurrence is lower than that reported in the study by Barton et al., ${ }^{7}$ whose rate of recurrence was approximately $13 \%$. Such difference may be explained by the fact that the study mentioned above, which included the same age group, used a higher propranolol dose.

Based on these data, it may be inferred that therapeutic success is related to the dose used. It is worth noting that, historically, digoxin has been the drug of choice due to the extensive successful experience in health centers worldwide. However, the majority of the most representative comparative studies between both drugs have clarified that propranolol use has increased whereas digoxin use has decreased. It has been speculated that this may be due to the lower incidence of reported adverse events with the use of this beta-blocker without any other evidence than the experience of each site because, comparatively, differences in SVT recurrence prevention and severe adverse event onset are not statistically significant. ${ }^{2,4,8}$ In 2017, Bolin et al., published a study in a neonatal population to compare both drugs. That study showed a greater survival for propranolol with a low rate of recurrence (similar to the one described in this study), without mortality during followup. ${ }^{9}$ In relation to on-treatment tachycardia recurrence, based on the Kaplan-Meier Table, it may be inferred that most events occur in the first month after diagnosis. Based on this, it may be speculated that, if the initial treatment response is adequate, the possibility of delayed events decreases.

Unlike other previously published studies that showed an association between preexcitation in the baseline ECG and a higher SVT recurrence, ${ }^{7}$ no statistically significant differences have been demonstrated in this population. This finding questions the need to consider other antiarrhythmics in an early manner only based on the presence of ventricular preexcitation.

This group of patients included some individuals with CHD who did not show higher recurrence rates. The study by $\mathrm{Chu}$ et al., ${ }^{2}$ which included patients with heart conditions similar to those reported in this study, did not find statistically significant differences between both groups.

In terms of adverse events, 2 patients had severe symptomatic adverse events, including hypotonia, pallor, and sensory deficiency with confirmed hypoglycemia, which led to hospitalization and drug discontinuation. It is worth noting that both patients developed their side effects in the setting of viral infections, which may have been the reason for their condition. The studies that used propranolol did not document this adverse event, but did observe bradycardia and hypotension more frequently., ${ }^{4,-10}$

The limitations of this study were the same as those related to retrospective studies, such as adverse event underestimation or masking by other conditions. This was not a study of pharmacokinetic and pharmacodynamic profiles, so the exact dose to obtain optimal results cannot be determined; therefore, it is necessary to conduct a randomized study to that end. In addition, this study did not establish a difference in terms of patient status during the onset of the first event (hospitalized or not, mechanical ventilation requirement, presence of catheters, inotrope requirement, and others), which may have affected the occurrence of SVT.

\section{CONCLUSION}

Propranolol prevented SVT recurrence in $70 \%$ of patients. Drug discontinuation was required in 2 patients due to severe adverse events (symptomatic hypoglycemia).

\section{REFERENCES}

1. Gándara-RicardoJA, Santander-Bohórquez D, Mora-Pabón G, Amaris-Peña O. Taquiarrtimias supraventriculares: Estado del arte. Rev Fac Med. 2016; 64(1):111-21.

2. Chu P, Hill K, Clark R, Smith B, et al. Treatment of supraventricular tachycardia in infants: analysis of a large multicenter database. Early Hum Dev. 2015; 91(6):345-50.

3. Moffet B, Salvin J, Kim J. Pediatric Cardiac Intensive Care Society 2014 Consensus Stamen: Pharmacotherapies in cardiac critical care antiarrhythmics. Pediatr Crit Care Med. 2016; 17(3 Suppl 1):S49-58. 
4. Sanatani S, Potts JE, Reed JH, Saul P, et al. The Study of Antiarrhythmic Medications in Infancy (SAMIS). A Multicenter, Randomized Controlled Trial Comparing the Efficacy and Safety of Digoxin Versus Propranolol for Prophylaxis of Supraventricular Tachycardia in Infants. Circ Arrhythm Electrophysiol. 2012; 5(5):984-91.

5. Wong KK, Potts JE, Etheridge SP, Sanatani S. Medications used to manage supraventricular tachycardia in the infant: A North American Survey. Pediatr Cardiol. 2006; 27(2):199203.

6. Seslar SP, Garrison MM, Larison C, Salerno JC. A multi-institutional analysis of inpatient treatment for supraventricular tachycardia in newborns and Infants. Pediatr Cardiol. 2013; 34(2):408-14.
7. Barton AL, Moffet BS, Valdes SO, Miyake C, et al. Efficacy and safety of high-dose propranolol for the management of infant supraventricular tachyarrythmias. J Pediatr. 2015; 166(1):115-8.

8. HornikC,ChuP,LiJ,ClarkR, etal.Comparativeeffectiveness of digoxin and propranolol for supraventricular tachycardia in infants. Pediatr Crit Care Med. 2014; 15(9):839-45.

9. Bolin EH, Lang SM, Tang X, Collins RT. Propranolol versus digoxin in the neonate for supraventricular tachycardia (from the Pediatric Health Information System). Am J Cardiol. 2017; 119(10):1605-10.

10. Kwon E, Jaachim S, Siegel D, Drolet B, et al. Retrospective review of adverse effects from propranolol in infants. JAMA Dermatol. 2013; 149(4):484-5. 